\title{
Deciphering the EU clinical trials regulation
}

\section{To the editor:}

The European Union (EU; Brussels) was founded to not only unite European countries economically and politically, but also to improve collaborations among member states. Academic clinical research has been part of these collaborations, and efforts have been made to harmonize the rules governing human testing. These efforts have led to the current European Clinical Trial Directive 2001/20/EC, which represents an important step toward achieving harmonized requirements for conducting clinical trials. However, the Directive has been criticized in academic circles, especially in relation to its fitness for the needs of academia vis-àvis pharmaceutical industry [1], the increased regulatory costs and complexity of the procedures for academic researchers. In April of 2014, the European Parliament and Council adopted the new Clinical Trial Regulation (EU) No. 536/2014 [2], with the hope of increasing the number of clinical trials conducted in the EU. It is worth noting that, the experienced gained from the Voluntary Harmonization Procedure (VHP), which was introduced in March 2009 by the Heads of Medicines Agencies' (HMA) Clinical Trial Facilitation Group (CTFG), has paved the way for the new regulation [3]. The new regulation will replace the current Directive, providing a directly applicable legal act throughout the EU. It also offers new principles and provisions that aim to counteract the limitations of working with the Directive, promote better harmonization and increase transparency in the reporting of clinical trial results (see Table 1). We discuss here some of these provisions, their possible impact on clinical research practice in general and the biotech sector in particular, and probable barriers that may limit their full potential.

The Regulation contains the definition of the new clinical trials sub-category "low intervention clinical trials". These are defined as trials on authorized medicinal products, used in accordance with the Summary of Product Characteristics (SmPC) or published evidence on their use, and where the additional procedures of the trial introduce no more than minimal risk to subject safety compared with the risk encountered in normal clinical practice. Currently, this activity is poorly regulated and is carried out by academics through the planning of comparative effectiveness trials to generate combination evidence-based medical therapies. This sub-category is expected to stimulate greater research on authorized products and to guide academic research 
into the more appropriate use of medicines. However, marketed drugs, especially newly approved ones, may not necessarily have the well-established safety profile required for such trials. Guidance regarding how to define minimal risk imposed by a trial based on already available post-marketing safety data may be required. Sponsors and investigators should also ensure that the data obtained aims to be clinically meaningful.

Other major changes introduced in the regulation especially for clinical trials with investigational medicinal products can be grouped into four categories: first, submission of clinical trial applications (CTA); second, safety reporting during the course of a clinical trial; third, global oversight and monitoring of clinical trials; and fourth, reporting data once a trial is completed and data submission for marketing authorization applications. We discuss these categories in more detail below (for other changes see Table 1).

Currently, the CTA submission procedure for multi-state trials across the EU varies greatly between member states, sometimes leading to divergent assessments and at times additional bureaucratic workload. The new regulation requires the submission of a single application dossier for all 'concerned member states' within the EU, where the sponsor is intending to carry out a multicenter clinical trial through a single web-based portal (the EU portal). The assessment of a clinical trial application will proceed through a twofold procedure (Fig. 1), with the 'reporting member state' coordinating the initial assessment (Part 1), and each concerned member state carrying out a separate assessment covering national features (Part 2). This coordinated assessment aims to support research in rare diseases and global epidemics, innovative therapies and personalized treatment strategies, where multicenter, multi-state trials are needed to recruit enough patients [4]. Nevertheless, to achieve these objectives, the national regulatory authorities need to ensure that they have the scientific expertise and staffing to assure a timely assessment.

In relation to safety reporting during the course of a clinical trial, the new regulation requires sponsors to submit their safety reports (SUSARs or 'suspected unexpected serious adverse reactions; and Annual Safety Reports) directly to an extended module of the Eudravigilance system. This will replace the current national reporting system, where sponsors are required to submit safety data to competent authorities and ethics committees of the individual member states. In addition to the previously mentioned safety reports, the new regulation states that only adverse events which influence the benefit-risk balance of the trial 
should be reported. However, guidance for adequately defining how a safety signal introduces a shift in the risk-benefit balance of a trial and what measures to use to easily quantify and monitor such a shift may be necessary. The perception of what impacts benefit or risk may differ between regulatory agencies versus industry or academia.

The regulation also aims to address challenges arising from the globalization of clinical research. There has been a trend toward industry conducting clinical studies outside of the EU and the United States to emerging markets [5]. Reasons may include the lower regulatory burden, less-expensive trial sites and the ability to quickly recruit the required patient numbers. The new regulation states that clinical trials conducted outside the EU to support marketing authorization applications in the EU must comply with principles equivalent to those set out in the EU regulation (e.g., good manufacturing practice (GMP) and good clinical practice (GCP) standards) and are subject to inspection by Concerned Member States. For a more effective longterm strategy, it may be useful to initiate a dialog on drug policy reform with developing countries and assist in establishing minimum eligibility requirements for multicenter trials.

International efforts are underway to improve transparency in reporting data once a clinical trial is completed, and reduce publication bias [6]. Within one year after the completion of each trial conducted under the regulation, both a summary of the results in structured format and a written summary in plain language, understandable to laypersons, must be submitted by the sponsor to the publicly accessible EU database. The regulation also requires Marketing Authorization Holders to submit the clinical study reports within 30 days after obtaining marketing authorization, disapproval or withdrawal of the application. The period specified by the new regulation aims to provide the sponsor with exclusive access to the information gained, allowing for the filing of additional patent protection and providing a competitive leverage for commercial sponsors. Moreover, the European Medicines Agency (EMA; London) has recently agreed to change its policy on pro-active publication of clinical trial data [7], making all clinical study reports included in Marketing Authorization Applications submitted to EMA after 1 January 2015 accessible to the public and useable for academic and non-commercial research purposes.

The regulation has recognized the complexity of developing biotech-derived pharmaceuticals. The period of the assessment of a CTA by a reporting member state may extend up to 50 days if the clinical trial involves an advanced therapy or biological medicinal products. 
However, sponsors will not gain access to this advantage and they will be requested to report any required additional information to the Reporting Member State (for Part 1) or Concerned Member State (for Part 2) within 12 days (Fig. 1). As a result, small biotech companies will be challenged by a mounting pressure to meet these deadlines. Most importantly, academic institutions - which are becoming integral players in the translation of biomedical technologies - will have to adapt their research efforts and resources. On a separate note, the new portal may have to take into account more specialized clinical trials such as those investigating advanced therapies, which require special measures for follow-up of efficacy and adverse reactions [8].

The regulation also touches upon several critical aspects that will influence the progress of the biomedical field. For instance, increasing the level of transparency in reporting clinical trial results will definitely help in reducing waste in biomedical research [9]. The centralized system for assessing CTA will promote academic biomedical research and innovation to address real unmet need, especially through partnering with other institutions in initiating multi-state trials. Although the new regulation had addressed some of the key challenges encountered in clinical research, at the same time, new ones are beginning to unfold. As an example, due to the rapid progress in the development of genome editing technologies, there is a clear need to initiate early dialog between regulators and scientists investigating various aspects of therapeutic genome editing as these technologies move into clinical trials [10]. It is worth noting that regulatory agencies such as Medicines \& Healthcare products Regulatory Agency (MHRA) are ready to meet these challenges, for example, by founding innovation offices that can help initiate early scientific advice and identify potential obstacles upfront. This could reduce the chance of finding deficiencies during the assessment of data presented in a CTA.

The application of the regulation is contingent upon the existence of a well-functioning EU portal and Database. An independent audit of these two is scheduled for August 2017 according to a timeline endorsed by the EMA Management Board on December 2015 [11]. A positive result, which shows the system has obtained full functionality in keeping with agreed upon functional specifications [12] will enable the regulation to commence operation by October 2018 at the latest. Subsequently, a three years transition period will start from the date of its application.

In summary, the new Regulation represents another major step toward improving the 
EU's clinical research environment. However, there may be certain barriers that still need to be addressed by regulators as well as by sponsors. These efforts may, in turn, put more pressure on regulatory agencies that need to be adequately prepared. Given the speed at which novel biotechnologies are advancing toward clinical testing, regulatory guidance will need to continue to evolve, and regulatory agencies have to have effective horizon-scanning exercises in place in order to be able to prepare for upcoming scientific evolution from early on. In addition, due to the higher level of transparency in reporting clinical outcomes, the scientific and ethical aspects of clinical trials are expected to be under public scrutiny. To that end, the continuation of a constructive discussion and open scientific debate on regulations to advance clinical research is encouraged, not only in Europe, but also worldwide - for the benefit of patients.

\section{Disclaimer}

The views expressed in this article are the personal views of the authors and may not be understood or quoted as being made on behalf of or reflecting the position of the National Institute for Biological Standards and Control (NIBSC), the Medicines \& Healthcare products Regulatory Agency (MHRA), or the European Medicines Agency (EMA) or any of its committees or working parties.

\section{Acknowledgment:}

We gratefully thank colleagues from the EMA, the MHRA and the Danish Health Medicines Agency for their valuable comments on earlier drafts of the manuscript.

\section{Mohamed Abou-El-Enein ${ }^{1} \&$ Christian K. Schneider ${ }^{2}$}

${ }^{1}$ Berlin-Brandenburg Center for Regenerative Therapies (BCRT), Charité University Medicine, Campus Virchow, Berlin, Germany

${ }^{2}$ National Institute for Biological Standards and Control (NIBSC), Medicines and Healthcare Products Regulatory Agency, South Mimms, Hertfordshire, United Kingdom and Twincore Centre for Experimental and Clinical Infection Research, Feodor-Lynen-Str. 7, 30625 Hannover, Germany

E-mail:mohamed.abou-el-enein@charite.de 
[1] L. J. Frewer, D. Coles, K. Champion, J. Demotes-Mainard, N. Goetbuget, K. Ihrig, I. Klingmann, C. Kubiak, S. A. Lejeune, F. McDonald, and J. Apperley, "Has the European Clinical Trials Directive been a success?," BMJ, vol. 340, p. c1862, Jan. 2010.

[2] Official J Eur Union, "Regulation (EC) No 536/2014 of the European Parliament and of the Council on clinical trials on medicinal products for human use, and repealing Directive 2001/20/EC. L 158."

[3] P. Hunter, "The health of European medical research. Attempts are under way to update EU regulations, with the aim of harmonizing clinical research across the continent.," EMBO Rep., vol. 12, no. 2, pp. 110-2, Feb. 2011.

[4] M. Abou-El-Enein, G. Bauer, P. Reinke, M. Renner, and C. K. Schneider, “A roadmap toward clinical translation of genetically-modified stem cells for treatment of HIV.," Trends Mol. Med., vol. 20, no. 11, pp. 632-642, Nov. 2014.

[5] S. W. Glickman, J. G. McHutchison, E. D. Peterson, C. B. Cairns, R. A. Harrington, R. M. Califf, and K. A. Schulman, "Ethical and scientific implications of the globalization of clinical research.," N. Engl. J. Med., vol. 360, no. 8, pp. 816-23, Feb. 2009.

[6] M. M. Mello, J. K. Francer, M. Wilenzick, P. Teden, B. E. Bierer, and M. Barnes, "Preparing for responsible sharing of clinical trial data.," N. Engl. J. Med., vol. 369, no. 17, pp. 1651-8, Oct. 2013.

[7] European Medicines Agency (EMA), "Publication of clinical reports," 2014. [Online]. Available:

http://www.ema.europa.eu/docs/en_GB/document_library/Press_release/2014/10/WC500 174767.pdf.

[8] C. K. Schneider, P. Salmikangas, B. Jilma, B. Flamion, L. R. Todorova, A. Paphitou, I. Haunerova, T. Maimets, J.-H. Trouvin, E. Flory, A. Tsiftsoglou, B. Sarkadi, K. Gudmundsson, M. O'Donovan, G. Migliaccio, J. Ancāns, R. Maciulaitis, J.-L. Robert, A. Samuel, J. H. Ovelgönne, M. Hystad, A. M. Fal, B. S. Lima, A. S. Moraru, P. Turcáni, R. Zorec, S. Ruiz, L. Akerblom, G. Narayanan, A. Kent, F. Bignami, J. G. Dickson, D. Niederwieser, M.-A. Figuerola-Santos, I. G. Reischl, C. Beuneu, R. Georgiev, M. Vassiliou, A. Pychova, M. Clausen, T. Methuen, S. Lucas, M. Schüssler-Lenz, V. Kokkas, Z. Buzás, N. MacAleenan, M. C. Galli, A. Linē, J. Gulbinovic, G. Berchem, M. Fraczek, M. Menezes-Ferreira, N. Vilceanu, M. Hrubisko, P. Marinko, M. Timón, W. Cheng, G. A. Crosbie, N. Meade, M. L. di Paola, T. VandenDriessche, P. Ljungman, L. D’Apote, O. Oliver-Diaz, I. Büttel, and P. Celis, "Challenges with advanced therapy medicinal products and how to meet them.," Nat. Rev. Drug Discov., vol. 9, no. 3, pp. 195-201, Mar. 2010.

[9] J. J. Meerpohl, L. K. Schell, D. Bassler, S. Gallus, J. Kleijnen, M. Kulig, C. La Vecchia, A. Marušić, P. Ravaud, A. Reis, C. Schmucker, D. Strech, G. Urrútia, E. Wager, G. Antes, and OPEN project consortium, "Evidence-informed recommendations to reduce 
dissemination bias in clinical research: conclusions from the OPEN (Overcome failure to Publish nEgative fiNdings) project based on an international consensus meeting.," $B M J$ Open, vol. 5, no. 5, p. e006666, 2015.

[10] J. Corrigan-Curay, M. O’Reilly, D. B. Kohn, P. M. Cannon, G. Bao, F. D. Bushman, D. Carroll, T. Cathomen, J. K. Joung, D. Roth, M. Sadelain, A. M. Scharenberg, C. von Kalle, F. Zhang, R. Jambou, E. Rosenthal, M. Hassani, A. Singh, and M. H. Porteus, "Genome Editing Technologies: Defining a Path to Clinic," Mol. Ther., vol. 23, no. 5, pp. 796-806, 2015.

[11] EMA.

http://www.ema.europa.eu/docs/en_GB/document_library/Other/2015/12/WC500199078. pdf (17 December 2015).

[12] EMA. (http://www.ema.europa.eu/docs/en_GB/document_library/Other/2014/12/WC500179339. pdf (25 March 2015). 